\title{
Functional renormalization group approach to the four-body problem
}

\author{
R. Schmidt ${ }^{1, a}$ and S. Moroz ${ }^{2}$ \\ 1 Physik Department, Technische Universität München, James-Franck-Strasse, D-85748 Garching, Germany \\ 2 Institut für Theoretische Physik, Universität Heidelberg, Philosophenweg 16, D-69120 Heidelberg, Germany
}

\begin{abstract}
We present a renormalization group analysis of the non-relativistic four-boson problem by means of a simple model with pointlike three- and four-body interactions. We investigate in particular the unitarity point where the scattering length is infinite and all energies are at the atom threshold. We find that the four-body problem behaves truly universally, independent of any four-body parameter confirming the findings of Platter et al. and von Stecher et al. [1-3].
\end{abstract}

\section{Introduction}

In recent years few-body physics experienced a renewed interest due to the advent of experiments with ultracold atomic gases. Whereas the study of few-body physics in nuclear systems is hindered by the large complexity of the interparticle potentials, the interactions in ultracold atomic gases are describable to high accuracy with very simple short-range models. In addition, ultracold atomic gases become even more attractive as an ideal theoretical and experimental playground since they do not only offer excellent experimental control but also the amazing possibility of tuning the two-body interaction strength, given by the swave scattering length $a$, over a wide range using so-called Feshbach resonances [4].

This made it possible to test central predictions of universality in few-body systems exhibiting strong interactions. Besides the universal two-body bound state, the weakly bound dimer, which obeys an universal formula for its binding energy, $E_{D}=\hbar^{2} /\left(m a^{2}\right)$ as $a$ approaches large values, about thirty years ago V. Efimov predicted also universal behavior [5] of the three-body system. The Efimov effect constitutes itself in the existence of infinitely many trimer states for infinitely large scattering length where the two-body interaction is just on the verge of having a bound state. The energy levels form a geometric spectrum and the three-body system is found to be universal in the sense that apart from the s-wave scattering length $a$ only one piece of information about the three-body system enters in the form of a so-called three-body parameter [6]. Recently, ultracold atom experiments made it possible to test Efimov's seminal predictions. First evidence in favor of the presence of these states had been found in the remarkable experiment by Kraemer et al. in 2006 [7]. The findings of Kraemer et al. stimulated extensive activity in the field of three-body physics, both experimentally [8-12] and theoretically; for recent reviews on also the latter see $[6,13]$ and references

\footnotetext{
a e-mail: richard.schmidt@ph.tum.de
}

therein. As a result, the Efimov effect in three-body systems is a well-understood phenomenon today.

The next natural step is to raise the question what the physics of four interacting particles may be. In their pioneering work, Platter and Hammer, et al. [1,2] investigated the four-body problem using effective interaction potentials and made the conjecture that the four-boson system exhibits universal behavior. They also found that no fourbody parameter is needed for a self-consistent renormalization of the theory. Calculating the energy spectrum of the lowest bound states in dependence on the scattering length $a$ the existence of two tetramer (four-body bound) states associated with each trimer was conjectured.

Recently, von Stecher, D'Incao, and Greene [3,14] investigated the four-body problem. They found that the Efimov trimer and tetramer states always appear as sets of states with two tetramers associated with each of the trimer levels and calculated the bound state energy spectrum of the lowest few sets of states. The calculation suggests that the energy levels within one set of states are related to each other by universal ratios, which were obtained from the behavior of these lowest sets of states. In accordance with the results of Platter et al. [1,2] the absence of any fourbody parameter was also demonstrated. In order to find experimental evidence of the tetramer states extremely precise measurements are required. Remarkably, Ferlaino et al. were able to observe signatures of the lowest two of the tetramer states in a recent experiment [15].

While the calculations by Platter et al. [1,2] and von Stecher et al. $[3,14]$ rely on quantum mechanical approaches, we want to shed light onto the four-body problem from a renormalization group (RG) perspective, complementary to the previous approaches.

Of special interest is the further investigation of universality in the four-body system. In this context the socalled unitarity point is of particular importance. Here all bound states are degenerate with the atom threshold, the scattering length $a$ is infinite, and physics becomes truly universal. The unitarity point is therefore the most interest- 


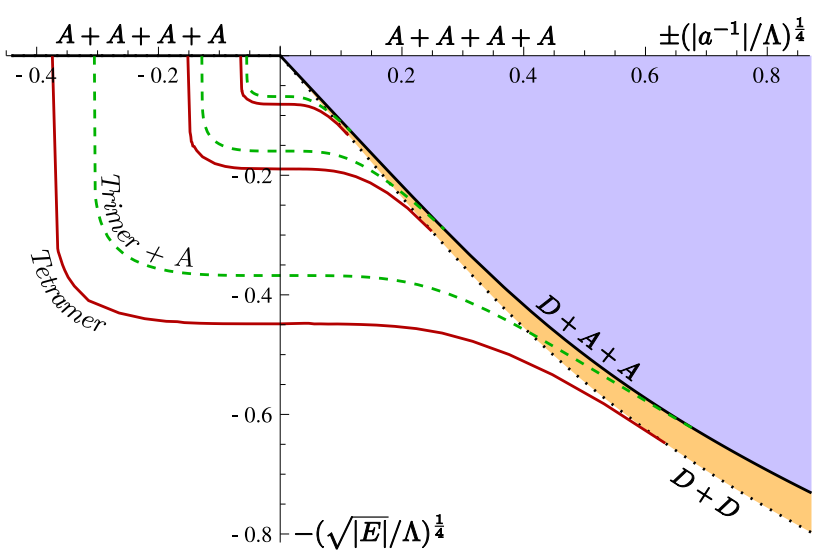

Fig. 1. (Color online) The generalized Efimov plot for four identical bosons. We plot the energy levels of the various bound states as a function of the inverse s-wave scattering length $a$ as numerically calculated in our approximative, effective theory. In order to improve the visibility of the energy levels we rescale both the dimensionless energy $E / \Lambda^{2}$ and the dimensionless inverse scattering length $a^{-1} / \Lambda$ where $\Lambda$ denotes the UV cutoff of our model. Also, we only show the first three sets of Efimov levels. The solid black line denotes the atom-atom-dimer threshold, while the dotted black line gives the dimer-dimer threshold. In the three-body sector one finds the well known spectrum of infinitely many Efimov trimer states (green, dashed) which accumulate at the unitarity point $E_{\psi}=a^{-1}=0$. In our pointlike approximation the four-body sector features a single tetramer (red, solid) associated with each trimer state.

ing one from a theory point of view. Unfortunately, it had been inaccessible with the previous calculations. Within our approach this point can easily be addressed. We investigate the renormalization group behavior of the relevant four-body interactions with an approximate, but simple and physically intuitive model which allows only for pointlike three- and four-body interactions. In the threebody problem universality manifests itself in an RG limit cycle of the three-body coupling. We find that this threebody limit cycle leads in turn to a "self-sustained" limit cycle of the four-body sector leaving no room for any fourbody parameter.

The RG method allows furthermore for computations away from the unitarity point. We calculate the bound state energy spectrum in the pointlike approximation (see Fig. 1) and investigate how the relations between tetramer and trimer states approach the universal limit as one comes closer to the unitarity point.

\section{Method and Model}

We are interested in the computation of the few-body properties, such as the bound state spectrum, of four identical bosons. In a quantum field theory approach the information about these properties can be extracted from the effective action $\Gamma$ which is the generating functional of oneparticle irreducible vertex functions $\Gamma^{(n)}$. As such it contains all information about a given system. For example, the existence of bound states is signaled by poles in the vertex functions corresponding to the relevant scattering processes. However, the computation of $\Gamma$ is a very complicated task as quantum fluctuations have to be integrated out on all length and therefore momentum scales $q$. In order to cope with this task we rely on the functional renormalization group [17] (for reviews see e. g. [18, 19]). The central quantity of the FRG is a scale dependent effective action functional, the so-called effective flowing action $\Gamma_{k}$. The effective flowing action $\Gamma_{k}$, which includes all fluctuations with momenta $q \gtrsim k$, interpolates between the classical action $S$ at some ultraviolet (UV) cutoff scale $k=\Lambda$ and the full quantum effective action $\Gamma$ in the limit $k \rightarrow 0$. The underlying idea is similar to Wilson's idea of momentum shell-wise integration of fluctuations. The evolution of $\Gamma_{k}$ is governed by the Wetterich equation [17], which is an exact, non-perturbative RG equation. It reads

$$
\partial_{k} \Gamma_{k}=\frac{1}{2} \operatorname{Tr}\left[\left(\Gamma_{k}^{(2)}+R_{k}\right)^{-1} \partial_{k} R_{k}\right],
$$

where $\Gamma_{k}^{(2)}$ is the flowing, full inverse propagator and the trace Tr sums over momentum $\mathbf{q}$ and Matsubara frequency $q_{0}$ as well as the internal degrees of freedom such as species of fields. The dependence on the RG scale $k$ is introduced by the regulator $R_{k}$. At the UV scale $k=\Lambda$ the effective flowing action $\Gamma_{k}$ equals the classical action $S$ and as we want to consider dilute atomic gases the UV scale $\Lambda$ is set to be of the order of the inverse Bohr radius $a_{0}^{-1}$. For most problems, quantum and statistical fluctuations will generate infinitely many terms in $\Gamma_{k}$. Due to this fact it is in practice impossible to solve Eq. (1) exactly. Therefore one has to decide for a truncation of $\Gamma_{k}$, which in turn corresponds to solving the theory only approximately.

In this work we choose the following truncation for the Euclidean flowing action, given by a simple two-channel model,

$$
\begin{aligned}
\Gamma_{k} & =\int_{x}\left\{\psi^{*}\left(\partial_{\tau}-\Delta+E_{\psi}\right) \psi+\phi^{*}\left[A_{\phi}\left(\partial_{\tau}-\frac{\Delta}{2}\right)+m_{\phi}^{2}\right] \phi\right. \\
& +\frac{h}{2}\left(\phi^{*} \psi \psi+\phi \psi^{*} \psi^{*}\right)+\lambda_{A D} \phi^{*} \psi^{*} \phi \psi+\lambda_{\phi}\left(\phi^{*} \phi\right)^{2} \\
& \left.+\beta\left(\phi^{*} \phi^{*} \phi \psi \psi+\phi \phi \phi^{*} \psi^{*} \psi^{*}\right)+\gamma \phi^{*} \psi^{*} \psi^{*} \phi \psi \psi\right\},
\end{aligned}
$$

where $\Delta$ denotes the Laplace operator and we use the natural, non-relativistic convention $2 M=\hbar=1$ with the atom mass $M . \psi$ denotes the field of the elementary bosonic atom, while the dimer, the bosonic bound state consisting of two elementary atoms, is represented by the field $\phi \sim \psi \psi$. Both the atom and the dimer field are supplemented with non-relativistic propagators with energy gaps $E_{\psi}$ and $m_{\phi}^{2}$, respectively. In our approximation the fundamental four-boson interaction $\sim \lambda_{\psi}\left(\psi^{*} \psi\right)^{2}$ is mediated by a dimer exchange, which yields $\lambda_{\psi}=-h^{2} / m_{\phi}^{2}$ in the limit of pointlike two-body interactions. The dynamical propagator of the dimer field $\phi$ with the wave function renormalization $A_{\phi}$ allows us to capture essential details of the momentum dependence of the two-body interaction. The only nonzero interaction, present at the microscopic UV scale $k=\Lambda$, is taken to be the Yukawa-type term with the coupling $h$. 


\section{$19^{\text {th }}$ International IUPAP Conference on Few-Body Problems in Physics}

The atom-dimer interaction $\lambda_{A D}$ as well as the various fourbody interactions $\lambda_{\phi}, \beta$, and $\gamma$ vanish at the UV scale and are built up via quantum fluctuations during the RG flow. In the general case of nonzero density and temperature one works in the Matsubara formalism and the integral in Eq. (2) sums over homogenous three-dimensional space and over imaginary time $\int_{x}=\int d^{3} x \int_{0}^{1 / T} d \tau$. Although our method allows us to tackle a full, many-body problem at finite temperature in this way, we are interested solely in the few-body (vacuum) physics in this paper, for which density $n$ and temperature $T$ vanish. For $T=0, \int_{x}$ reduces to an integral over infinite space and time. Our truncation (2) is based on the simple structure of the non-relativistic vacuum and, as demonstrated in $[16,20]$, numerous simplifications occur when solving Eq. (1) compared with the general, many-body case.

We note that the flowing action (2) has a global $U(1)$ symmetry which corresponds to particle number conservation. In the vacuum limit it is also invariant under spacetime Galilei transformations which restricts the form of the nonrelativistic propagators to be functions of $\partial_{\tau}-\Delta$ for the atoms and $\partial_{\tau}-\Delta / 2$ for the dimers. All couplings present in Eq. (2) are allowed to flow during the RG evolution and are taken to be momentum-independent in Fourier space. Besides the ansatz for $\Gamma_{k}$ we must choose a suitable regulator function $R_{k}$ in order to solve the flow equation (1). Based on our recent treatment of the closely related threefermion problem $[21,22]$, we choose the regulators,

$$
\begin{aligned}
& R_{\psi}=\left(k^{2}-\mathbf{p}^{2}\right) \theta\left(k^{2}-\mathbf{p}^{2}\right), \\
& R_{\phi}=\frac{A_{\phi}}{2}\left(k^{2}-\mathbf{p}^{2}\right) \theta\left(k^{2}-\mathbf{p}^{2}\right) .
\end{aligned}
$$

These regulators are optimized in the sense of $[19,23]$ and allow to obtain analytical results.

\section{Two-body sector}

A remarkable and very useful feature of the vacuum flow equations is comprised by a special hierarchy: the flow equations of the $N$-body sector do not influence the renormalization group flows of the lower $N-1$-body sector [16]. For this reason the different $N$-body sectors can be solved subsequently. In this spirit we first solve the two-body sector, then investigate the three-body sector in order to finally approach the four-body problem ${ }^{1}$.

The solution for the two-body sector can be found analytically $[21,24]$. The only running couplings in the twobody sector are the dimer gap $m_{\phi}^{2}$ and the wave function renormalization $A_{\phi}$ and the flow equations of the two-body sector in terms of Feynman diagrams are shown in Fig. 2. As there are no possible nonzero diagrams for the Yukawa coupling $h$, it does not flow in the vacuum limit.

The infrared (IR) values of the couplings $h$ and $m_{\phi}^{2}$ can be related to the low-energy s-wave scattering length $a$ via

$$
a=-\frac{h^{2}(k=0)}{16 \pi m_{\phi}^{2}\left(k=0, E_{\psi}=0\right)} .
$$

1 The atom inverse propagator (one-body sector) is not renormalized in the non-relativistic vacuum.
Knowing the analytical solution of the two-body sector, this relation can be used to fix the initial values of our model. For the UV value of the dimer gap $m_{\phi}^{2}$ we find

$$
m_{\phi}^{2}(\Lambda)=-\frac{h^{2}}{16 \pi} a^{-1}+\frac{h^{2}}{12 \pi^{2}} \Lambda+2 E_{\psi}
$$

The first term fixes the s-wave scattering length according to Eq. (4), while the second term represents a counterterm taking care of the UV renormalization of the two-body sector. Finally, the last term accounts for the fact that the dimer consists of two elementary atoms. Additionally, we choose $A_{\phi}(\Lambda)=1$ which corresponds to the effective range $r_{\text {eff }}=-\frac{64 \pi}{h^{2}}$.

In the limit of large, positive scattering length there exists a universal, weakly bound dimer state. In order to find its binding energy we calculate the pole of the dimer propagator, corresponding to the condition $m_{\phi}^{2}\left(E_{\psi}, k=0\right)=0$, which yields in the limit $E_{\psi} / \Lambda^{2} \ll 1$

$$
\begin{aligned}
E_{D}=-2 E_{\psi} & =-2\left(\frac{h^{2}}{64 \pi}-\sqrt{\frac{h^{4}}{(64 \pi)^{2}}+\frac{h^{2} a^{-1}}{32 \pi}}\right)^{2} \\
& =-\frac{2}{r_{\text {eff }}^{2}}\left(1-\sqrt{1-\frac{2 r_{\text {eff }}}{a}}\right)^{2} .
\end{aligned}
$$

In the limit $h \rightarrow \infty$, corresponding to $r_{\text {eff }} \rightarrow 0$ one recovers the well-known result $E_{D}=-2 / a^{2}$. The dimer bound state energy is shown as a function of the inverse scattering length in Fig. 1 (black solid line). The deviation from the universal $\sim 1 / a^{2}$ scaling for large inverse scattering lengths is due to the finite size of $h$ which is taken to be $h^{2} / \Lambda=10$ in Fig. 1. In the regime of small scattering length $a$ one finds a crossover of the different behavior of the dimer binding energy which then has the limiting behavior $E_{D}=4 /\left(a r_{\text {eff }}\right)$.

\section{Three-body sector}

The bound state spectrum of the three-body sector is much richer than the one of the two-body system. Due to the Efimov effect an infinite tower of three-body bound (trimer) states appears as one approaches the so-called unitarity limit $E_{\psi}=a^{-1}=0$. Remarkably, trimer states exist even for negative scattering lengths $a$ where no two-body bound state is present; they become degenerate with the atom threshold for negative scattering length and merge into the atom-dimer threshold for positive $a$. An additional threebody parameter in needed in order to determine the actual positions of the degeneracies [25]. In our truncation, the three-body sector contains a single, contact $\phi^{*} \psi^{*} \phi \psi$ term with a coupling $\lambda_{A D}$, which is assumed to vanish in the UV. It is built up by quantum fluctuations during the RG flow and the corresponding Feynman diagrams of the flow equation for $\lambda_{A D}$ are shown in Fig. 2. For the unitarity point, $E_{\psi}=a^{-1}=0$ we are able to obtain an analytical solution for the flow equation of $\lambda_{A D}$. In this limit all intrinsic 
length scales drop out of the problem and the system becomes classically scale invariant. At unitarity, the Yukawa coupling $h$ is dimensionless and the only (extrinsic) length scale present is the inverse ultraviolet cutoff $\Lambda^{-1}$, which defines the validity limit of our effective theory.

In our approximation the dimer field $\phi$ develops a large anomalous dimension $\eta=-\frac{\partial_{t} A_{\phi}}{\bar{A}_{\phi}}=1$ at unitarity which is consistent with the exact solution of the two-body sector $[16,20]$. As the atom and dimer propagators have vanishing gaps in the IR the two-body sector respects a continuous scaling symmetry.

In order to find the solution of the three-body sector we switch to the rescaled, dimensionless coupling $\tilde{\lambda}_{A D} \equiv$ $\frac{k^{2}}{h^{2}} \lambda_{A D}$. One finds that the flow equation for $\tilde{\lambda}_{A D}$ becomes independent of $k$ and $h$,

$$
\partial_{t} \tilde{\lambda}_{A D}=a \tilde{\lambda}_{A D}^{2}+b \tilde{\lambda}_{A D}+c, \quad a, b, c \in \mathbb{R} .
$$

The numerical coefficients $a, b$, and $c$ can be found in [24], and as demonstrated in $[16,26]$, the behavior of the solution of this type of flow equation is determined by the sign of the discriminant $D$ of the right hand side of Eq. (7) which is $D=b^{2}-4 a c<0$. The solution of Eq. (7) is given by

$$
\tilde{\lambda}_{A D}(t)=\frac{-b+\sqrt{-D} \tan \left(\frac{\sqrt{-D}}{2}(t+\delta)\right)}{2 a},
$$

where $\delta$ is connected to the three-body parameter and determines the initial condition. Most remarkably, the threebody sector undergoes a quantum anomaly: The RG flow of the renormalized coupling $\tilde{\lambda}_{A D}$ exhibits a limit cycle, which, due to its periodicity, breaks the classically continuous scaling symmetry to the discrete subgroup $Z$. The Efimov parameter can be determined from the period of the limit cycle [16] leading in our approximation to

$$
s_{0}=\frac{\sqrt{-D}}{2} \approx 0.925203 .
$$

The exact result is given by $s_{0} \approx 1.00624$ [6]. Considering the simplicity of our pointlike approximation the agreement is quite good. In fact, in previous work [16] we have shown how to obtain the exact value of $s_{0}$ using the FRG.

The presence of N-body bound states leads to divergencies in the RG flow of the corresponding N-body vertices. The periodic divergencies in the analytical solution of $\tilde{\lambda}_{A D}$ in Eq. (8) correspond therefore to the presence of the infinitely many Efimov trimer states at the unitarity point.

We can use the latter identification to calculate the bound state spectrum also away from unitarity. The trimer binding energies are calculated by determining the atom energies $E_{\psi}$ for which $\tilde{\lambda}_{A D}$ exhibits divergencies in the IR as function of $a^{-1}$. The trimer binding energy is then given by $E_{T}=-3 E_{\psi}$. The result is shown in Fig. 1 .

In the unitarity limit the trimer binding energies form a geometric spectrum and the ratio between adjacent levels is given by

$$
\frac{E_{T}^{(n+1)}}{E_{T}^{(n)}}=e^{-\frac{2 \pi}{s_{0}}},
$$
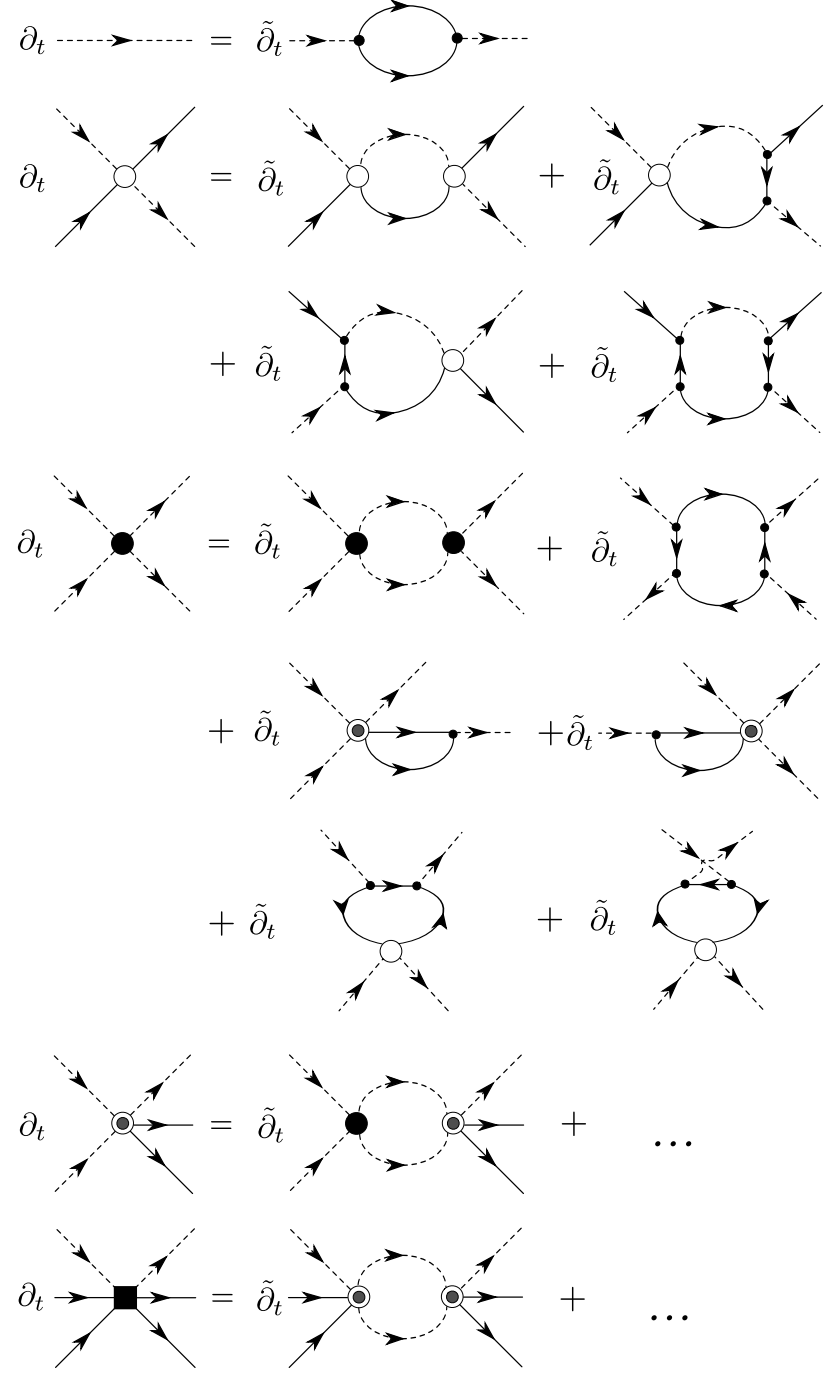

Fig. 2. The flow equations in terms of Feynman diagrams. All internal lines denote full, regularized propagators. The scale derivative $\tilde{\partial}_{t}$ on the right hand side of the flow equations acts only on the regulators. The vertices are: Yukawa coupling $h$ (small black dot), atom-dimer vertex $\lambda_{A D}$ (open circle), dimer-dimer coupling $\lambda_{\phi}$ (black circle), coupling $\beta$ (two circles), and the atom-atom-dimer vertex $\gamma$ (black square). Due to the large number of diagrams for the latter two vertex functions, we only show two exemplary diagrams.

which can be understood from the limit cycle flow of $\lambda_{A D}$. At each scale $k=\Lambda e^{t}$, where $\lambda_{A D}$ diverges, one hits a trimer state. The RG scale $k$ can in turn be connected to the atom energy $E_{\psi}[16,21,22]$ and as the divergencies appear periodically in $t$ one easily obtains Eq. (10).

There is an additional universal relation obeyed by the trimer energy levels which we may take as a measure of the quality of our approximation. It is given as the relation between the trimer binding energy $E^{*}$ for $a \rightarrow \infty$ and value of $a$ for which the trimer becomes degenerate with the atom-dimer $\left(a_{+}^{*}\right)$ and three-atom threshold $\left(a_{-}^{*}\right)$, respectively. For comparison we define a wave number $\kappa^{*}$ by $E^{*}=-\hbar^{2} \kappa^{* 2} / M$ (in our convention, $E^{*}=-2 \kappa^{* 2}$ ) and 


\section{$19^{\text {th }}$ International IUPAP Conference on Few-Body Problems in Physics}

find

$$
a_{+}^{*} \kappa^{*} \approx 0.08, \quad a_{-}^{*} \kappa^{*} \approx-1.68
$$

which has to be compared with the exact result $a_{+}^{*} \kappa^{*}=$ $0.07076, a_{-}^{*} \kappa^{*}=-1.56(5)$ from the fully momentumdependent calculation in $[6,25]$. The agreement with our approximate solution suggests that our model should provide a solid basis for the step to the four-body problem.

\section{Four-body sector}

Recently, the solution of the four-body problem in the lowenergy limit has gained a lot of interest. In quantum mechanical calculations the existence of two tetramer (fourbody bound) states was conjectured for each of the infinitely many Efimov trimers $[1,2]$. By calculating the lowest few sets of bound state levels von Stecher et al. [3,14] concluded that both ratios of energies between the different tetramers and the trimer state approach universal constants. However, with the quantum mechanical approach the calculation directly at the unitarity point $\left(a^{-1}=E_{\psi}=0\right)$ turns out to be difficult, although this point is of great interest when one wants to gather evidence for universality of the four-body system. In fact, in the three-body sector the infinite RG limit cycle appears only exactly at the unitarity point and it is directly connected to the breaking of the continuous scale symmetry. Within our approach, the unitarity region is easily accessible.

In order to investigate the four-body sector we include all possible, U(1) symmetric, momentum-independent interaction couplings in the effective flowing action $\Gamma_{k}$. Like in the three-body sector we assume all these couplings to be zero at the microscopic UV scale $\Lambda$. Under this condition one can easily show that only the three four-body couplings $\lambda_{\phi}, \beta$, and $\gamma$ are built up by quantum fluctuations and are therefore included in Eq. (2). All other couplings will stay zero during the whole RG evolution.

For the investigation of the unitarity limit we work with rescaled, dimensionless couplings

$$
\tilde{\lambda}_{\phi}=\frac{k^{3}}{h^{4}} \lambda_{\phi}, \quad \tilde{\beta}=\frac{k^{4}}{h^{3}} \beta, \quad \tilde{\gamma}=\frac{k^{5}}{h^{2}} \gamma .
$$

By the use of the rescaled couplings we find three coupled ordinary differential equations, which are again coupled to the two- and three-body sectors, but become explicitly independent of $h$ and $k$. We show the diagrammatic representation of the flow equations in Fig. 2. Their computation is straightforward, but as the analytical expressions become extremely lengthy we refrain from showing them here.

In order to determine the bound state spectrum of the four-boson system we exploit the fact that the appearance of bound states is connected with divergent vertex functions $\Gamma_{k}^{(n)}$. At this point we must note that these infinities are complicated to handle in a numerical solution of the theory. In particular, the numerical treatment of unbounded limit cycles is problematic due to the periodic infinities during the RG flow. In order to circumvent this difficulty we use the method of complex extension, developed in [26]. The basic idea is to extend the domain of the running couplings to the complex plane

$$
\begin{aligned}
\lambda_{A D} \rightarrow \lambda_{A D, 1}+i \lambda_{A D, 2} & \lambda_{\phi} \rightarrow \lambda_{\phi, 1}+i \lambda_{\phi, 2} \\
\beta \rightarrow \beta_{1}+i \beta_{2} & \gamma \rightarrow \gamma_{1}+i \gamma_{2} .
\end{aligned}
$$

On the one hand this effectively doubles the number of real flow equations and additional initial conditions must be provided. We choose $\lambda_{A D, 2}=\epsilon=10^{-11}$ in our numerical calculation and take all other imaginary parts to be zero in the UV. On the other hand this procedure allows us to perform the numerical integration of the flow equations as it regularizes the periodic infinities in the flow and makes the numerical treatment feasible. Physically, by the complex extension we convert the stable bound states into metastable resonances and by taking different values of $\epsilon$ we are able to vary the decay width of the resonances. One may compare this with the procedure of Braaten and Hammer [27] who introduce a parameter $\eta^{*}$ in order to model the decay of the trimers to deeply bound states which have not been included in the effective model. In this line we also view our complex extension as a way to include these deeply bound states in the FRG calculation. Specifically, we find that for $\epsilon \ll 1$ the decay width of the $\mathrm{n}^{\text {th }}$ Efimov trimer $\Gamma_{\mathrm{T}}^{(n)}$ is given by $\Gamma_{\mathrm{T}}^{(n)}=4 \epsilon E_{\mathrm{T}}^{(n)}$ at unitarity. This is in agreement with the result in [6]

$$
\Gamma_{\mathrm{T}}^{(n)} \approx \frac{4 \eta^{*}}{s_{0}} E_{\mathrm{T}}^{(n)}
$$

which holds for small $\eta^{*}$. Thus, for $\epsilon \ll 1$, the relation to the parameter $\eta^{*}$ introduced by Braaten and Hammer is given by

$$
\epsilon=\frac{\eta^{*}}{s_{0}}
$$

The result of the numerical calculation of the four-body sector at unitarity is shown in Fig. 3. Here, we display the RG flows of the real parts of all nonzero three- and four-body sector couplings. In the lower part of the figure we show the flow over a wide range of values of the $R G$ scale $t=\ln (k / \Lambda)$ while in the upper part we show the RG flow in more detail for appearance of the first few resonances. The three-body coupling $\tilde{\lambda}_{A D, 1}$ (black dotted line) exhibits the well-known limit cycle behavior, described in Section 4, with the period being connected to the Efimov parameter $s_{0}$. Remarkably, there is an additional limit cycle in the flow of the four-body sector couplings with a periodic structure of exactly the same frequency as the threebody sector. This four-body sector limit cycle exhibits resonances which are shifted with respect to the ones of the three-body system. The magnitude of this shift is given by a new universal number, which is inherent to the four-body sector.

Our observation is that the four-body sector is intimately connected with the three-body sector in the unitarity limit. It is permanently attached to the running of the three-body sector from the first three-body resonance on. From here 


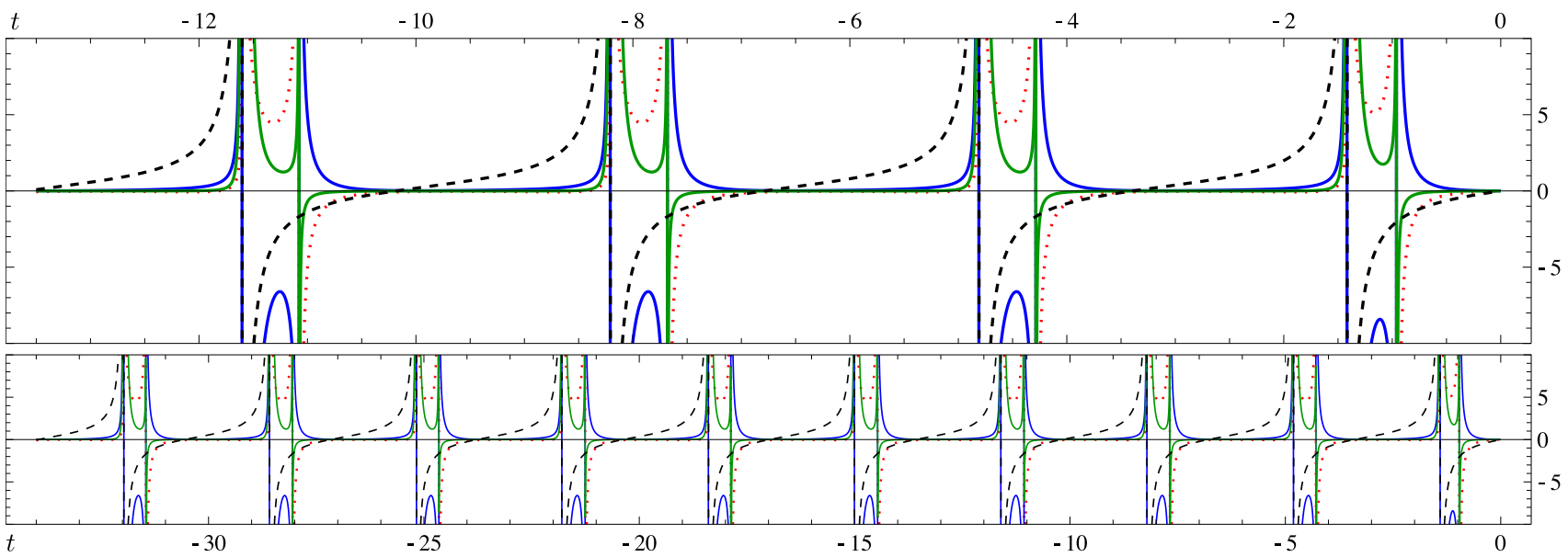

Fig. 3. (Color online) Renormalization group limit cycle behavior of the three- and four-body sector at the unitarity point $E_{\psi}=a^{-1}=0$. The real parts of the couplings are plotted as functions of $t=\ln (k / \Lambda)$. Not only the three-body coupling $\lambda_{A D, 1}$ (dashed, black) exhibits a limit cycle behavior, but also the four-body sector couplings $\lambda_{\phi, 1}$ (red, dotted), $\beta_{1}$ (blue, solid), and $\gamma_{1}$ (green, solid) obey a limit cycle attached to the three-body sector with the same period.

on the periodic structure of the flow remains unchanged as one goes to smaller values of $k$. Due to this tight bond between the three- and four-body sector, there stays no room for an additional four-body parameter.

We also find that the magnitude of the shift beyond the first resonance is neither dependent on the initial values of the four-body sector couplings in the UV nor is it influenced by finite range corrections which we are able to check by choosing different values for the Yukawa coupling $h$. Arbitrary choices lead to the same behavior. Having done this calculation directly in the unitarity limit our conclusion is, that, within our simple approximation, the four-body sector behaves truly universal and independent of any four-body parameter confirming the conjecture made by Platter et al. and von Stecher et al.. We expect that universality will also hold for an improved truncation.

Naively one expects that each resonance in the flow of the vertex functions is connected to the presence of a bound state. As one observes there are also additional resonances in the four-body sector being degenerate with the threebody sector resonances. However, we arrive at the conclusion that these resonances are artifacts of our approximation. The mathematical structure of the flow equations is of a kind that divergencies in the three-body sector directly lead to a divergent four-body sector. We are confident that the resonances at these positions will disappear as one includes further momentum dependencies in the field theoretical model. Therefore we can already infer from the calculation at unitarity that within our approximation we are only able to resolve a single tetramer state attached to each trimer state also away from unitarity. In contrast, the "exact" quantum mechanical calculations in $[1-3,14]$ predict the existence of two tetramer states which have recently been observed by Ferlaino et al. [15]. As one includes further momentum dependencies, we may speculate however that not only the degenerate resonance disappears but also new, genuine resonances associated with the "missing" tetramer state will appear at the same time.
Such an effect is known from field theoretical studies of the three-body problem. Here, it is essential to include a momentum dependent atom-atom interaction. Only under this condition one arrives at the quadratic equation as in (7) and observes the Efimov effect.

We can also use our model to investigate the full bound state energy spectrum by solving the flow equations for arbitrary values of the scattering length $a$. The energy levels of the various bound states are then determined by varying the energy of the fundamental atoms $E_{\psi}$ such that one finds a resonant four-body coupling in the IR. The result of this calculation is shown in Fig. 1, where we plot the energy levels of the various bound states versus the inverse scattering length. We find one tetramer state attached to each of the Efimov trimer states. These tetramer states become degenerate with the four-atom threshold for negative scattering length and merge into the dimer-dimer threshold for positive $a$. In the experiment this leads to the measured resonance peaks in the four-body loss coefficient. In order not to overload the plot we show only the first three sets of levels, although the FRG method allows to calculate an arbitrary number of them. One also observes that the shape of the tetramer levels follows the shape of the trimer levels. In analogy to the three-body sector one can calculate a universal formula relating a tetramer binding energy $E_{T e t}^{*}=-2 \kappa_{T}^{* 2}$ at $a \rightarrow \infty$ with the corresponding scattering length at which the tetramer becomes degenerate with the four-atom threshold $a_{T-}^{*}$ and the dimer-dimer threshold $a_{T+}^{*}$, respectively. We find

$$
a_{T-}^{*} \kappa_{T}^{*} \approx-1.75, \quad a_{T+}^{*} \kappa_{T}^{*} \approx 0.20 .
$$

In their recent quantum mechanical calculations von Stecher et al. were able to calculate the lowest few sets of bound state energy levels [3]. From their behavior it was inferred that the ratio between the tetramer and trimer binding energies approaches a universal number within these first few sets of levels. Figuratively speaking it is therefore expected that the universal regime in the energy plot in Fig. 1 is reached very fast as one goes to smaller $a^{-1}$ and $E_{\psi}$. 
$19^{\text {th }}$ International IUPAP Conference on Few-Body Problems in Physics
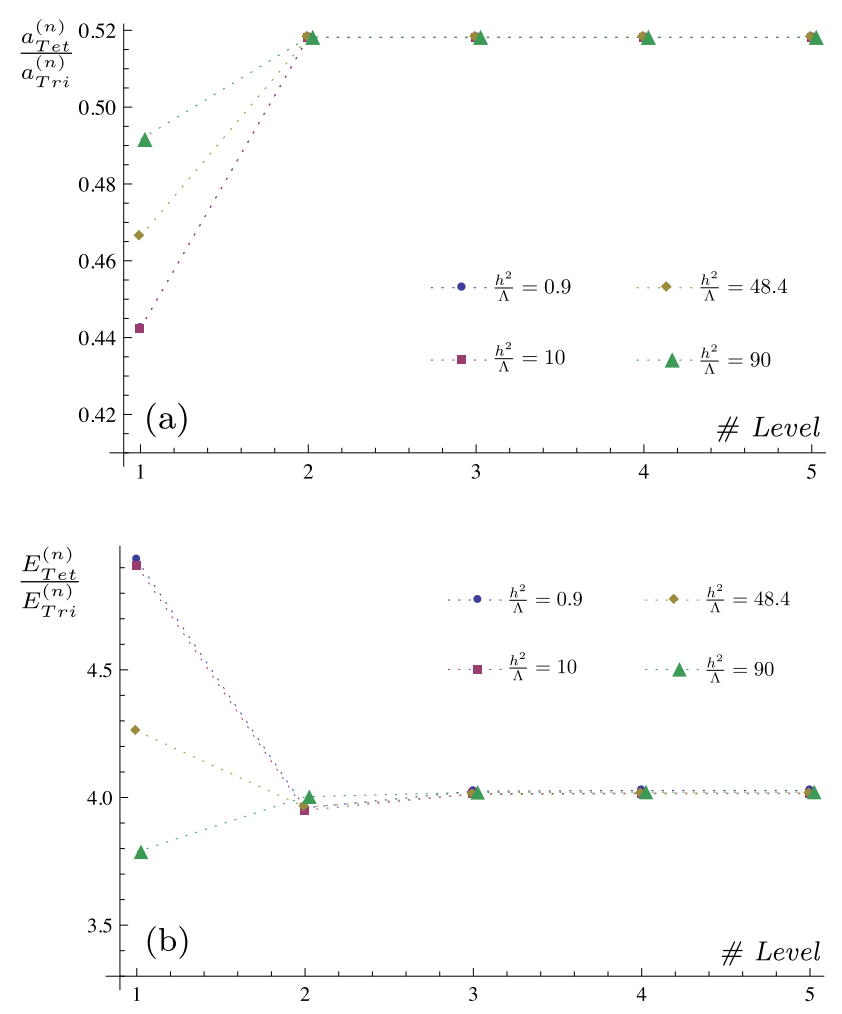

Fig. 4. (Color online) Calculation of the universal ratios for the lowest five set of levels. The calculation is done for different values of the Yukawa coupling $h^{2} / \Lambda$ which determines the effective range in our model. The dotted lines are only guide for the eye. (a) Ratios between the values of scattering lengths $a_{T e t}^{(n)}$ and $a_{T r i}^{(n)}$ for which the tetramer and corresponding trimer become degenerate with the four-atom threshold. (b) Ratios between the values of binding energies $E_{T e t}^{(n)}$ and $E_{T r i}^{(n)}$ at resonance $a \rightarrow \infty$.

In order to investigate this observation we calculate the behavior of two ratios as a function of the set of level for which they are determined. The first ratio relates the negative scattering lengths $a_{T e t}^{(n)}$ and $a_{T r i}^{(n)}$ for which the $\mathrm{n}^{\text {th }}$ tetramer and trimer become degenerate with the atom threshold. The second is the ratio between the binding energies of the $\mathrm{n}^{\text {th }}$ tetramer $E_{T e t}^{(n)}$ and the $\mathrm{n}^{\text {th }}$ trimer $E_{T r i}^{(n)}$ at resonance, $a \rightarrow \infty$. The resulting plots are shown in Fig. 4. We calculate the ratios for different values of the microscopic couplings in order to test the degree of universality of the various sets of energy levels. In the plots we show in particular the dependence on the choice of the Yukawa coupling $h$ determining the effective range $r_{\text {eff }}$ of the model. As one sees, only the first of the ratios depend on the microscopic details. Already from the second set of levels on the microscopic details are washed out and the ratios become independent of the choice of initial conditions: The regime of universality is reached extremely fast and as $a^{-1}$ and $E_{\psi}$ are lowered one will ultimately find the four-body limit cycle described above.

For the asymptotic ratios we find

$$
a_{T e t}^{(n)} \approx 0.518 a_{T r i}^{(n)},
$$

$$
E_{T e t}^{(n)} \approx 4.017 E_{\text {Tri }}^{(n)}
$$

Von Stecher et al. find $a_{T e t}^{(n)} / a_{T r i}^{(n)} \approx 0.43(0.9)$ for the deeper (shallower) bound tetramer and $E_{T e t}^{(n)} / E_{T r i}^{(n)} \approx 4.58(1.01)$, respectively. Considering the simplicity of our model the agreement is quite good.

With an ultracold bosonic Cs gas Ferlaino et al. found $a_{T e t}^{(n)} / a_{T r i}^{(n)} \approx 0.47(0.84)$. In this experiment only the lowest set of tetramer states in the energy spectrum had been accessible due to the particular scattering length profile. Considering our observation that the deepest set of levels is still strongly dependent on the microscopical details it cannot be expected to find the universal numbers in this particular setting. Therefore more experiments for bosons interacting via a larger scattering lengths would be desirable.

\section{Conclusions}

In this work, we investigated the four-body problem with the help of the functional renormalization group. Employing a simple two-channel model with pointlike three- and four-body interaction we were able to investigate universal properties at the unitarity point $a \rightarrow \infty, E_{\psi}=0$ as well as to perform computations away from it.

In the RG language the Efimov physics of the three-body problem manifests itself as an infinite RG limit cycle behavior of the three-body coupling constant at unitarity. We found that also the four-body sector is governed by such a limit cycle which is solely induced by the RG running of the three-body sector, signaling the absence of a four-body parameter.

We also computed the energy spectrum away from unitarity and were able to obtain the universal relations between four- and three-body observables in our approximation. Our calculation provides an explanation for the findings of von Stecher et al. [3], who found that these ratios approach universal constants very quickly as they are computed for higher and higher excited states. We also found a dependence of the ratios for the lowest level on microscopic details such as the effective range. This in turn is of relevance for the experimental observations by Ferlaino et al. [15]. In this experiment the lowest states have been measured and one can therefore not expect to find the exact universal relations between them.

Considering the simplicity of our model, the agreement with the previous studies in [1-3] is quite good. There had been some disagreement in literature about universality and the absence or existence of a four-body parameter, see e.g. [28-30]. Our RG results support the conclusion that the four-body system is universal and independent of any four-body parameter.

An important shortcoming of the pointlike approximation is the absence of the shallower of the two tetramer states. Obviously the pointlike approximation of the three- and four-body sectors is not sufficient and in future work one should include momentum dependent interactions. From the energy spectrum in Fig. 1 it becomes also evident that 
the excited tetramer states can decay into an energetically lower lying trimer plus atom. The higher excited states in the four-body system are therefore expected to have an intrinsic finite decay width [2]. Whether this width has a universal character still remains an open question as well as in which way the corresponding imaginary coupling constants will change the RG analysis.

The inclusion of the full momentum dependencies in the three- and four-body sector seems to be a rather complicated task. In the effective field theory study of the threeboson system the introduction of a dynamical dimer field, often called the di-atom trick [6], has been a decisive step towards the exact solution of the three-body problem. From this perspective we suggest that the inclusion of a dynamical trimer field in the effective action might help to simplify the momentum dependent calculation.

The four boson system remains still a subject with many open questions. With our RG analysis in the pointlike approximation, we made the first step towards a renormalization group description of the four-body problem supplementing the previous quantum mechanical approaches. From this perspective this work provides a starting point for a deeper understanding of universality in the four-body problem.

Acknowledgements - RS is supported by the DFG within the FOR 801 'Strong correlations in multiflavor ultracold quantum gases'. SM is grateful to KTF for support.

\section{References}

1. L. Platter, H.-W. Hammer, and Ulf-G. Meißner, Phys. Rev. A 70, 052101 (2004).

2. H.-W. Hammer, and L. Platter, Eur. Phys. J. A 32, 113 (2007).

3. J. von Stecher, J. P. D'Incao, and C. H. Greene, Nature Phys. 5, 417-421 (2009).

4. C. Chin, R. Grimm, P. Julienne, and E. Tiesinga, arXiv:0812.1496 (2008).

5. V. Efimov, Phys. Lett. B 33, 563 (1970); V. Efimov, Nucl. Phys. A 210, 157 (1973).

6. E. Braaten, H.W. Hammer, Phys. Rept. 428, 259 (2006).

7. T. Kraemer et al., Nature 440, 315 (2006).

8. S. Knoop et al., Nature Phys. 5, 277 (2009).

9. T. B. Ottenstein et al., Phys. Rev. Lett. 101, 203202 (2008).

10. J. H. Huckans et al., Phys. Rev. Lett. 102, 165302 (2009); J. R. Williams et al., arXiv:0908.0789v1 (2009).

11. M. Zaccanti et al., Nature Phys. 5, 586 (2009).

12. G. Barontini et al. Phys. Rev. Lett. 103, 043201(2009).

13. L. Platter, arXiv:0904.2227 (2009).

14. J. P. D'Incao, J. von Stecher, and C. H. Greene, Phys. Rev. Lett. 103, 033004 (2009).

15. F. Ferlaino, et al., Phys. Rev. Lett. 102, 140401 (2009).

16. S. Moroz, S. Floerchinger, R. Schmidt, and C. Wetterich, Phys. Rev. A 79, 042705 (2009).

17. C. Wetterich, Phys. Lett. B 301, 90 (1993).
18. K. I. Aoki, Int. J. Mod. Phys. B 14, 1249 (2000); C. Bagnuls, C. Bervillier, Phys. Rept. 348, 91 (2001); M. Salmhofer and C. Honerkamp, Prog. Theor. Phys. 105, 1 (2001); C. Wetterich, Int. J. Mod. Phys. A 16, 1951 (2001); J. Berges, N. Tetradis, and C. Wetterich, Phys. Rept. 363, 223 (2002); W. Metzner, Prog. Theor. Phys. Suppl. 160, 58 (2005); B. Delamotte, e-print arXiv:cond-mat/0702365.

19. J. M. Pawlowski, Ann. Phys. 322, 2831 (2007).

20. S. Diehl, H. C. Krahl, M. Scherer, Phys. Rev. C 78, 034001 (2008).

21. S. Floerchinger, R. Schmidt, S. Moroz, and C. Wetterich, Phys. Rev. A 79, 013603 (2009).

22. S. Floerchinger, R. Schmidt, and C. Wetterich, Phys. Rev. A 79, 053633 (2009).

23. D. F. Litim, Phys. Lett. B 486, 92 (2000).

24. R. Schmidt, and S. Moroz, arXiv:0910.4586v1 (2009).

25. P. F. Bedaque, H. W. Hammer, and U. van Kolck, Phys. Rev. Lett. 82, 463 (1999); Nucl. Phys. A 646, 444 (1999).

26. S. Moroz, and R. Schmidt, Ann. Phys., doi:10.1016/j.aop.2009.10.002 (2009); arXiv:0909.3477 (2009).

27. E. Braaten, H.-W. Hammer, Phys. Rev. Lett. 87, 160407 (2001); Phys. Rev. A 70042706 (2004).

28. M. T. Yamashita, L. Tomio, A. Delfino, and T. Frederico, Europhys. Lett. 75, 555 (2006).

29. S. K. Adhikari, T. Frederico, and I. D. Goldmann, Phys. Rev. Lett. 74, 487 (1995).

30. R. D. Amado, and F. C. Greenwood, Phys. Rev. D 7, 2517 (1973). 